\title{
CORRELAÇÃO ENTRE A MEDIDA ANTROPOMÉTRICA DE COXA E A FORÇA EM 10 REPETIÇÕES MÁXIMAS
}

\author{
CORRELATION BETWEEN THIGH ANTHROPOMETRIC MEASUREMENT \\ AND STRENGTH IN 10 MAXIMUM REPETITIONS
}

\author{
Jurandir Silva, \\ Fernando Bessi, \\ Gabriel Guimar, \\ Giovanne Ribeiro, \\ Rodrigo Vale, \\ Rodolfo Nunes, \\ Vicente Lima
}

\section{Resumo}

Verificar se existe correlação entre a medida antropométrica de coxa com a força muscular em 10 repetições máximas (10RM) no exercício de cadeira extensora. Foram selecionadas 16 mulheres treinadas (idade: $20 \pm 6$ anos) para a mensuração dos dados antropométricos e avaliação da força de 10RM. Os avaliados foram orientados de forma padronizada para garantir maior uniformidade dos valores. Foram realizadas três tentativas a fim de se achar a carga de 10RM. Toda a coleta de dados foi realizada em um dia. Após a coleta dos dados, foram realizados os trabalhos estatísticos, os quais possibilitaram ver se os dados eram paramétricos ou não paramétricos. Com os resultados podese observar que não ocorreu correlação entre os valores de perímetro de coxa e força de $10 R M$, visto o valor $r=-0,2271$. Isso revela uma correlação moderada negativa de acordo com a tabela de correlação de Pearson. Pode-se concluir que não ocorreu correlação entre a perimetria de coxa e a força de 10RM, ou 


\section{Original}

\section{Atenção à saúde}

seja, a força na amostra ocorre não só por questões de espessura do segmento, e pode sofrer influência de outros fatores, como adaptação neural e composição corporal.

Palavras-chave: Perimetria de coxa, treinamento de força, mulher, $10 \mathrm{RM}$ repetições múltiplas.

\section{Abstract}

Check whether there is a correlation between anthropometric measurements of thigh muscle strength in 10 maximum repetitions (10RM) in the leg extension exercise. We selected 16 women (aged: $20 \pm 6$ years) for implementation of the PAR- Q, measurement of anthropometric data and evaluation of the strength in 10RM. Subjects were instructed in a standardized way to ensure greater uniformity of values. There were three attempts in order to find the 10RM load. All data collection was carried out in one day. After collecting the data, statistical work was done, allowing checking whether data was parametric or nonparametric. The results showed no correlation between the values of thigh perimeter and the strength in 10RM, since the $r=-0.2271$. It displays a moderate negative correlation in accordance with Pearson correlation table. It can be concluded that there was no correlation between the thigh circumference and strength in 10RM, that is, the strength on the sample occurs not only because in thickness issues of the segment, and may be influenced by other factors such as neural adaptation and body composition.

Keywords: Thigh perimetry, strength training, woman, multiple repetitions. 


\section{Introdução}

A prática regular de atividades físicas pode trazer benefícios a saúde. Dentre eles, pode-se destacar a melhora dos componentes da aptidão física, diminuição de dores articulares, aumento e manutenção da densidade mineral óssea, melhora da utilização de glicose, melhora do perfil lipídico e diminuição da resistência vascular ${ }^{1-3}$.

O treinamento de força (TF) é uma das formas mais conhecidas de exercícios físicos que pode ser usado para desenvolver o condicionamento físico de atletas e de não-atletas ${ }^{4}$. No TF é possível treinar a força máxima ${ }^{5}$, ou seja, a capacidade máxima que um músculo ou grupamento muscular possui de gerar tensão contra uma resistência. A força máxima é frequentemente medida pelo teste de uma repetição máxima (1RM) ou por repetições máximas $(R M)^{6-8}$.

O desenvolvimento da força motora provém de alguns fenômenos, como mecanismos de adaptações neurais ${ }^{9}$, que pode ser notado principalmente nas semanais iniciais de treinamento ${ }^{10}$. Após essas adaptações, o processo de hipertrofia muscular também se apresenta como uma possível adaptação ao $\mathrm{TF}^{11}$. A hipertrofia muscular caracteriza-se pelo aumento no tamanho das fibras musculares devido ao acúmulo de substâncias contráteis, actina e miosina, e de substâncias não contráteis, principalmente glicogênio e água, no sarcoplasma das fibras musculares ${ }^{12}$.

Embora existam, na literatura, estudos que associam ganhos de força com o ganho de hipertrofia muscular ${ }^{13,14}$, há relato na literatura em que os ganhos de força não foram acompanhados por respostas hipertróficas ${ }^{15}$. Diversos estudos buscaram verificar a associação da força muscular com a hipertrofia utilizando diferentes meios de avaliação. Dentre eles, destacam-se a ressonância magnética ${ }^{16}$, tomografia computadorizada ${ }^{16}$ e equação matemática. Contudo, informações sobre a relação entre medida antropométrica e a força muscular ainda são escassas na literatura. 


\section{Original}

\section{Atenção à saúde}

Sendo assim, é relevante analisar esse método de fácil utilização e baixo custo, podendo ser facilmente utilizado para medidas populacionais, podendo contribuir na avaliação e prescrição do profissional de Educação Física. Pelo exposto, o presente estudo possui como objetivo analisar a correlação entre a medida antropométrica de coxa com a força muscular obtida em 10 repetições máximas no exercício de cadeira extensora.

\section{Métodos}

\section{Amostra}

Foram selecionadas para participar do presente estudo 16 mulheres, com idade de $20 \pm 6$ anos e índice de massa corporal (IMC) de $22 \pm 4 \mathrm{~kg} / \mathrm{m}^{2}$, praticantes de musculação e estudantes do curso de Educação Física de uma universidade da zona oeste do município do Rio de Janeiro. Elas deveriam praticar musculação com uma frequência de pelo menos três vezes por semana há no mínimo seis meses. O estudo adotou como critérios de exclusão: a) apresentar PAR-Q positivo; b) apresentar dor articular ao realizar o teste; c) utilizar recursos ergogênicos.

O estudo foi realizado dentro das normas éticas previstas na resolução 466/2012 do Conselho Nacional de Saúde ${ }^{17}$ e todos os sujeitos assinaram um termo de consentimento livre e esclarecido.

\section{Procedimentos}

\section{Medida antropométrica de coxa}

Foi utilizada uma fita métrica metálica (Sanny ${ }^{\circledR}$, Brasil) para mensurar 0 perímetro médio da coxa, obtido através do ponto médio entre a prega inguinal e a borda superior da patela (ponto médio da coxa) ${ }^{18}$.

O avaliado ficou na posição bípede, relaxado, com os braços pendentes ou cruzados sobre o peito, os membros inferiores ligeiramente afastados e o peso igualmente distribuído pelos dois pés. O observador posicionou-se 


\section{Original}

\section{Atenção à saúde}

lateralmente em relação ao sujeito e colocou a fita métrica sobre a pele nua, ao nível do ponto mid-trocantérico-tibial lateral. Nesta posição pode-se fazer os ajustes necessários para que a fita ficasse horizontal em relação ao solo e perpendicular ao eixo longitudinal do membro inferior sem fazer muita pressão sobre os tecidos. A aferição foi repetida duas vezes para cada lado, a fim de se obter maior precisão na coleta dos dados. A média das circunferências das duas pernas foi utilizada ${ }^{19}$

\section{Teste da Força de 10 RM}

O teste foi realizado na cadeira extensora (Buick ${ }^{\circledR}$, Brasil). Foram realizados os ajustes necessários de modo que os joelhos do avaliado estivessem alinhados ao eixo de rotação do aparelho, as costas apoiadas no banco, o apoio do coxim apoiado acima do tornozelo e os joelhos posicionados formando um ângulo de $90^{\circ}$. Foi orientado ao avaliado realizar a extensão completa do joelho ${ }^{17}$. Foram fornecidas orientações sobre a técnica de execução do exercício antes do teste a fim de padronizar os movimentos durante o teste de 10 RM. Além disso, estímulos verbais foram realizados para manter o nível de estimulação elevado 20,21.

Antes da mensuração da carga máxima de $10 \mathrm{RM}$, os avaliados realizaram um aquecimento muscular, utilizando $50 \%$ da carga estipulada para a primeira tentativa de $10 \mathrm{RM}$. Os intervalos entre as tentativas para estabelecer as 10RM foram fixados em cinco minutos.

\section{Análise estatística}

Os dados foram analisados pelo pacote estatístico IBM SPSS Statistics 20 para Windows e apresentados de forma descritiva com a utilização de média, desvio padrão e valores mínimos e máximos. Para a verificação da normalidade dos dados aplicou-se o teste de Shapiro-Wilk. O teste de Correlação de Pearson foi empregado para analisar possíveis associações entre as variáveis do estudo.

\section{Resultados}


Os resultados do perímetro de coxa e sobrecarga do teste de 10RM estão apresentados na Tabela 1.

Tabela 1. Resultados de perímetro de coxa e sobrecarga

\begin{tabular}{ccc}
\hline & Perímetro de coxa & Sobrecarga 10RM \\
\hline Média & 58,17 & 73,33 \\
Desvio padrão & 3,66 & 25,35 \\
Mínimo & 54,00 & 45,00 \\
Máximo & 66,00 & 115,00 \\
\hline
\end{tabular}

Foi traçado o gráfico 1 de dispersão para verificar a correlação entre as variáveis dependentes. O gráfico mostra que não há uma correlação linear entre o perímetro de coxa e a sobrecarga de 10RM, visto que o valor de $r^{2}$ é 0,0516, mostrando que somente $5,16 \%$ dos dados analisados podem ser correlacionáveis.

Ao realizar o teste de Pearson para verificar o coeficiente de correlação linear, obtivemos o valor $r$ de -0,2271, mostrando uma correlação moderada negativa de acordo com a tabela de correlação de Pearson. 


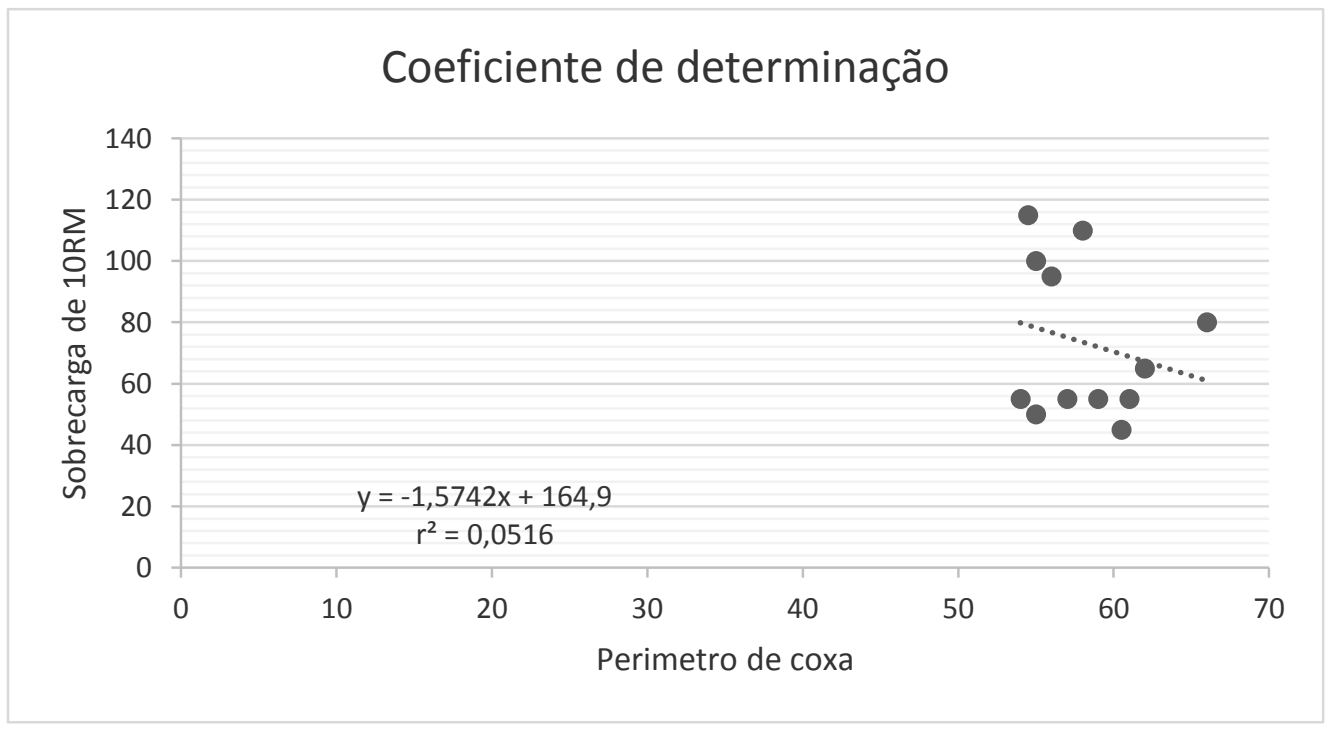

Gráfico 1. Relação entre perímetro de coxa e a sobrecarga de 10RM

\section{Discussão}

Com a mensuração das variáveis antropométricas e a força muscular no teste de 10RM, pode-se observar uma correlação fraca negativa, mostrando que nesse estudo não foi observado uma correlação significativa entre a perimetria de coxa e a força de 10RM. Tal fato pode ser explicados por diversos fatores, dentre eles as adaptações neurais e morfológicas que ocorrem com a prática do TF. Desse modo, pode-se destacar as adaptações neurais, que promovem um aumento significativo da força muscular, sem aumento da área de secção transversa do músculo, não modificando a perímetria ${ }^{4,22}$.

Bonganha et al. ${ }^{15}$ encontraram resultado semelhante quando avaliaram o comportamento da força muscular e a participação dos indicadores de hipertrofia nos ganhos de força, após 32 semanas de treinamento com pesos, prescrito por zona alvo de repetições máximas, em mulheres na pós-menopausa. Participaram 14 mulheres saudáveis e não ativas fisicamente. A hipertrofia muscular foi verificada através do perímetro do braço e da coxa. A força muscular foi avaliada pelo teste de uma repetição máxima nos exercícios leg 


\section{Original}

\section{Atenção à saúde}

press horizontal e rosca bíceps direta. Ao final de 32 semanas, houve aumento significativo nos níveis de força. No entanto, não foram notadas maiores modificações na hipertrofia muscular. Esses resultados reafirmam os pressupostos verificados no presente estudo que não verificou uma correlação entre o perímetro da coxa e força muscular.

Oliveira et al. ${ }^{23}$ analisaram a força isométrica e dinâmica e circunferência da coxa em sete mulheres adultas jovens iniciantes no TF com assimetrias laterais leve/moderadas das coxas. Após realizarem as medidas antropométricas, as participantes foram submetidas ao teste de força isométrica de membros inferiores, e ao teste de força dinâmica de $1 \mathrm{RM}$ de forma unilateral. Ao final dos testes, não foi observada diferença significativa para as medidas de circunferência e as forças isométrica e dinâmica, corroborando os resultados do presente estudo, que verificou não haver correlação entre a antropometria da coxa e a força muscular.

A determinação da área de secção transversa estimada (AST) pode ser usada para descrever populações de atletas e não-atletas, sendo fundamental, também, para avaliar os efeitos de várias mudanças que ocorrem no músculo esquelético, como por exemplo, a hipertrofia e a atrofia muscular ${ }^{24}$. Westphal et al. ${ }^{25}$ correlacionaram a massa corporal total, a massa corporal magra e a AST com a força máxima de sete mulheres com idade média de 33,86 $\pm 7,36$ anos, praticantes de TF há 1,93 \pm 0,45 anos, nos exercícios supino horizontal e leg press $45^{\circ}$. Verificou-se uma correlação significativa envolvendo a AST de braço. Tal resultado difere do presente estudo que não verificou correlação entre espessura muscular e produção de força. A utilização de metodologias diferentes podem explicar essa distinção de resultados.

Entre alguns fatores que auxiliam na hipertrofia muscular, pode-se citar a alimentação ${ }^{26}$, questões hormonais ${ }^{27} \mathrm{e} o$ tipo de treinamento ${ }^{28}$. Contudo, outro aspecto que pode influenciar na medida antropométrica de qualquer segmento do corpo humano é o acumulo de gordura em determinada região ${ }^{29}$. Sabe-se que as mulheres possuem, em geral, um percentual de gordura superior aos homens. Além disso, as diferenças de distribuição de massa magra entre homens e mulheres também podem influenciar a produção de força ${ }^{33}$. Ademais, 
a maior concentração da gordura corporal nas mulheres encontra-se nos membros inferiores, o que influencia negativamente a produção de força ${ }^{30}$.

Uma das razões para a ausência de correlação entre a perimetria de coxa e a força em 10RM no presente estudo, pode ser devido a maior concentração de gordura nos membros inferiores de mulheres. Outra razão para a ausência de significância destes resultados pode ter sido a utilização de uma amostra reduzida, o que pode ser considerado um fator limitante do estudo. O pequeno número amostral utilizado pode ter sido outro fator relacionado à ausência de resultados significativos entre as variáveis estudadas. Desse modo, devem ser realizados novos estudos com um maior número de sujeitos.

\section{Conclusões}

No presente estudo, não foi observada correlação entre a perimetria de coxa e a força em 10RM, indicando que os parâmetros investigados não se correlacionaram nos indivíduos estudados. Sendo assim, o perímetro da coxa não pode ser utilizado para a avaliação da força muscular.

Dessa forma, sugere-se que futuras pesquisas que avaliem a correlação de medidas antropométricas e força muscular utilizem outros parâmetros de avaliação, como diferentes instrumentos e protocolos, a fim de tornar os resultados mais fidedignos. Sugere-se, também, para estudos futuros, a utilização de métodos de diagnóstico por imagem, como, por exemplo, a ressonância magnética.

\section{Referências}

1. Brito AF, Santos SB, Oliveira CVC, Nóbrega TKS, Forjaz CLM, Santos ACS. High-intensity resistance exercise promotes postexercise hypotension greater than moderate intensity and affects cardiac autonomic responses in women who are hypertensive. J Strength Cond Res. 2015; 29(12): 3486-93.

2. Sposito AC, Caramelli B, Fonseca FAH, Bertolami MC, Afiune Neto A, Souza $A D$, et al. Sociedade Brasileira de Cardiologia. IV Diretriz brasileira sobre 


\section{Original}

\section{Atenção à saúde}

dislipidemias e prevenção da aterosclerose: Departamento de Aterosclerose da Sociedade Brasileira de Cardiologia. Arq Bras Cardiol. 2007; 88(1): 2-19.

3. Almeida JA, Balsamo S, Nascimento DC, Prestes J, Teixeira TG, Tibana RA, Voltarelli FA, Qualidade de vida e síndrome metabólica em mulheres brasileiras: análise da correlação com a aptidão aeróbia e a força muscular, 2015; 2(11): 4861.

4. Fleck SJ, Kraemer WJ. Fundamentos do treinamento de força: fisiologia neuromuscular e adaptações ao treinamento de força. 3. ed. Porto Alegre: Artmed; 2006.

5. Ploutz LL, Tesch PA, Biro RL, Dudley GA. Effect of resistance training on muscle use during exercise. J Appl Physiol. 1994; 76(4): 1675-81.

6. Batista GJ, Barbosa MTS. Efeitos de 10 semanas do treinamento com peso sobre indicadores da composição corporal em indivíduos do gênero masculino. Revista digital de Educação Física. Movimentum. 2006; 1: 224-70.

7. Cardoso FS, Curtolo M, Júnior IL, Natour J. Avaliação da qualidade de vida, força muscular e capacidade funcional em mulheres com fibromialgia. Rev Bras Reumatol. 2011; 51(4): 338-50.

8. Gentil P. Bases científicas do treinamento de hipertrofia. Adaptações morfológicas ao treinamento. 5. ed. São Paulo: Copyright; 2014.

9. Barroso R, Tricoli V, Ugrinowitsch C. Adaptações neurais e morfológicas ao treinamento de força com ações excêntricas. Rev Bras Ciênc Mov. 2005; 13(2): 111-122.

10. Moritani T, Vries HA. Neural factors versus hypertrophy in the time course of muscle strength gain. Am J Phys Med. 1979; 58: 115-130.

11. Assumpção CO, Leite RD, Neto JB, Pellegrinotti IL, Prestes J, Urtado CB. Efeito do treinamento de força periodizado sobre a composição corporal e aptidão física em mulheres idosas. Rev Edu Fís. 2008; (19):581-90.

12. Santarém JM. Musculação: princípios atualizados: fisiologia, treinamento e nutrição. São Paulo: Fitness Brasil; 1995.

13. Fett CA, Fett WCR. Correlação de parâmetros antropométricos e hormonais ao desenvolvimento da hipertrofia e força muscular. R. bras. Ci. e Mov. 2003; 11(4): 27-32. 


\section{Original}

\section{Atenção à saúde}

14. Spineti J. Comparação entre diferentes modelos de Periodização sobre a força e espessura Muscular em uma sequência dos menores Para os maiores grupamentos musculares. Rev Bras Med Esporte 2013; 19(4)

15. Bonganha V, Botelho RMO, Conceição MS, Chacon-Mikahil MPT, Madruga VA. Relações da força muscular com indicadores de hipertrofia após 32 semanas de treinamento com pesos em mulheres na pós-menopausa. Motricidade. 2010; 6(2): 23-33.

16. Housh DJ, Housh TJ, Weir JP, Weir LL, Johnson GO, Stout JR. Anthropometric estimation of thigh muscle cross-sectional area. Med Sci Sports Exerc 1995; 27(5): 784-791.

17. Conselho Nacional de Saúde (Brasil). Resolução no 466, de 12 de dezembro de 2012. Diário Oficial da União.2013; 12, p. 59.

18. Heyward VH, Stolarczyk LM. Avaliação da composição corporal aplicada. 2. ed. São Paulo: Manole; 2000.

20. Gordon C, Chunlea WC, Roche AF. Stature, recumbent length, and weight. In: Lohman TG, Roche AF, Martorell R, editors. Antropometric standardization reference manual. Champaign: Human Kinetics; 1988

21. Baechle TR, Earle RW. Essentials of strength training and conditioning. 3. ed. Champaign: Human Kinetics; 2000.

22. Garber CE, Blissmer B, Deschenes MR, Franklin BA, Lamonte MJ, Lee IM, et al. American College of Sports Medicine position stand. Quantity and quality of exercise for developing and maintaining cardiorespiratory, musculoskeletal, and neuromuscular fitness in apparently healthy adults: guidance for prescribing exercise. Med Sci Sports Exerc. 2011; 43(7): 1334-1359.

23. Oliveira LS, Gomes JRD, Souza DAN, Mendes JF, Crispiniano EC, Aniceto RR. Assimetrias de coxa não influenciam a força isométrica e dinâmica de mulheres adultas jovens. Revista Brasileira de Prescrição e Fisiologia do Exercício. 2016; 10(58): 247-253.

24. Housh DJ, Housh TJ, Weir JP, Weir LL, Johnson GO, Stout JR. Anthropometric estimation of thigh muscle cross-sectional. Medicine and Science in Sports and Exercise. 1995; 27(5): 784-791. 


\section{Original}

\section{Atenção à saúde}

25. Westphal M, Baptista RR, Oliveira AR. Relações entre massa corporal total, massa corporal magra, área de seção transversa e $1 \mathrm{RM}$ em mulheres. Revista Brasileira de Cineantropometria e Desempenho Humano. 2006; 8(1): 52-57.

26. Bacurau R. Nutrição e suplementação esportiva. São Paulo: Phorte; 2000.

27. Hakkinen K, Pakarinen A, Kraemer WJ, Häkkinen A, Valkeinen H, Alen M. Selective muscle hypertrophy, changes in EMG and force, and serum hormones during strength training in older women. J. Appl. Physiol. 2001; 91: 569-80.

28. Higbie EJ, Cureton KJ, Warren GL, Prior BM. Effects of concentric and eccentric training on muscle strength, cross-sectional area, and neural activation. J. Appl. Physiol. 1996; 81(5): 2173-2181.

29. Arruda MF, Simões MJS. Perfil do excesso de peso na infância e sua influência sobre o sistema musculoesquelético de escolares. Cinergis. 2007; 8(2): 37-47.

30. Castro MJ, McCann DJ, Shaffrath JD, Adams WC. Peak torque per unit crosssectional area differs between strength-trained and untrained young adults. Med Sci Sports Exerc. 1995; 27(3): 397-403. 